\title{
SOLVENT-FREE C-ALKYLATION OF BARBITURIC ACID IN THE NANOCRYSTALLINE MORDENITE MEDIA
}

\author{
GHOLAMHASSAN IMANZADEH, ${ }^{a, 1}$ SAEED KABIRI, ${ }^{a}$ SAEED TAGHAVI, ${ }^{a}$ MOHAMMADREZA \\ ZAMANLOO ${ }^{a}$ YAGOUB MANSOORI ${ }^{a}$
}

${ }^{a}$ Department of Chemistry, College of Science, University of Mohaghegh Ardabili 56199-11367, Ardabil, Iran

(Received: October 14, 2012 - Accepted: May 30, 2013)

\begin{abstract}
A simple, efficient and clean procedure has been developed for the $C$-alkylation of barbituric acid. The Michael addition of barbituric acid to $\alpha, \beta$-unsaturated esters by employing $\mathrm{K}_{2} \mathrm{CO}_{3}$ in the nano-mordenite media ( $\mathrm{K}_{2} \mathrm{CO}_{3}$ /nano-MOR), as catalyst, gave $C$-alkylated barbituric acid in good to excellent yield. Nano-MOR showed good reusability. $\mathrm{K}_{2} \mathrm{CO}_{3} /$ nano-MOR catalyst has been found to be an excellent catalyst for the reaction under solvent-free conditions at $80-90{ }^{\circ} \mathrm{C}$.
\end{abstract}

Keywords: Solvent-free, $C$-Alkylation, Barbituric Acid, $\alpha, \beta$-Unsaturated Esters, Nano-Mordenite (Nano-MOR),

\section{INTRODUCTION}

Barbiturates, that are derivatives of barbituric acid (pyrimidine-2,4,6trione), belong to one of the most important classes of drug molecules..$^{1-7}$ These compounds were first introduced for medical use in $1911 .{ }^{8}$ More than 2500 barbiturates have been synthesized and in the height of their popularity about 50 were marketed for human use. ${ }^{9}$ Today, only about a dozen are still in use. Although barbituric acid itself is devoid of biological activity, its derivatives such as single or double $C$-alkylated barbituric acids have sedative, hypnotic, anesthetic and anticonvulsant activities. ${ }^{10}$ Clinically important hypnotic-sedative barbiturates have substitutions at sites 1,2- and, especially, 5- of barbituric acid. ${ }^{11-13}$ Side chains at position 5- (especially one of them is branched) is essential for hypnotic activity. ${ }^{14}$

Diverse routes have been reported for the synthesis of $C$-alkylated derivatives of barbituric acid the majority of them involving a condensation of urea and malonic esters derivatives. ${ }^{15-18}$ But very little extent of work on the direct $C$-alkylation of barbituric acid is found in literature. Therefore, it is felt necessary to develop an efficient method for the performing of these reactions. In continuation of our studies on solvent-free organic reaction on solid supported reagents, ${ }^{19-21}$ we report herein an efficient procedure for the synthesis of $C$-alkylated barbituric acids via Michael addition of barbituric acid to $\alpha, \beta$-unsaturated esters in supported potassium carbonate on nano-MOR $\left(\mathrm{K}_{2} \mathrm{CO} /\right.$ nano-MOR) media under solvent-free conditions (Scheme 1). In this method, no dialkylation at position 5- was observed at all. To the best of our knowledge, there are only few reports on the addition reaction of barbituric acid on $\alpha, \beta$-unsaturated carbonyl compounds. ${ }^{22-24}$<smiles>[R]C[C@@H]1C[C@@H](C(=O)O[R])NC(=O)N1</smiles>

Scheme 1.

\section{RESULTS AND DISCUSSION}

The Michael addition of barbituric acid 1 to ethyl acrylate was investigated as model reaction in presence of $\mathrm{K}_{2} \mathrm{CO}_{3}$ /nano-MOR, in the absence of solvent. It was found that when a mixture of ethyl acrylate $(1 \mathrm{mmol})$, barbituric acid (1 mmol), and $\mathrm{K}_{2} \mathrm{CO}_{3}(1 \mathrm{mmol}) /$ nano-MOR $(0.5 \mathrm{gr})$ ) was kept at $80-90{ }^{\circ} \mathrm{C}$ for $2 \mathrm{~h}$ the corresponding mono- $C$-alkylated barbituric acid was obtained in $90 \%$ yield as the sole product. The double Michael addition on this substrate was not successful even with addition excess Michael acceptor ( $2 \mathrm{mmol}$ ethyl acrylate). Encouraged by these results we investigated the general applicability of this method on diverse $\alpha, \beta$-unsaturated esters (Table 1 ). The reactions were completed in 1-3.5 $\mathrm{h}$ and the desired mono- $C$-alkylated barbituric acids were produced in good to excellent yields (Table 1, entries 1-16). It was observed that the steric hindrance of Michael acceptor did not influence negatively the yield of products (Table 1, entries 6-16).

Table 1. C-Alkylation of barbituric acid in the presence and absence of nano-MOR under solvent-free conditions.

\begin{tabular}{|c|c|c|c|c|}
\hline Entry & Ester & Product & $\begin{array}{l}\text { Time } \\
(\mathrm{h})^{\mathrm{a}}\end{array}$ & $\begin{array}{l}\text { Yield } \\
(\%)^{\mathrm{b}}\end{array}$ \\
\hline 1 & & & $\begin{array}{l}17: 20 \\
(2: 00)\end{array}$ & $60(90)$ \\
\hline 2 & & & $\begin{array}{l}19: 45 \\
(2: 00)\end{array}$ & $55(87)$ \\
\hline 3 & & & $\begin{array}{l}19: 40 \\
(1: 55)\end{array}$ & $58(87)$ \\
\hline 4 & & & $\begin{array}{l}21: 30 \\
(2: 10)\end{array}$ & $55(85)$ \\
\hline 5 & & & $\begin{array}{l}24: 15 \\
(2: 15)\end{array}$ & $50(80)$ \\
\hline 6 & & & $\begin{array}{l}28: 35 \\
(3: 00)\end{array}$ & $48(78)$ \\
\hline 7 & & & $\begin{array}{l}24: 20 \\
(2: 30)\end{array}$ & 49 (79) \\
\hline 8 & & & $\begin{array}{l}20: 15 \\
(1: 50)\end{array}$ & $62(88)$ \\
\hline 9 & & & $\begin{array}{l}19: 30 \\
(2: 00)\end{array}$ & $64(89)$ \\
\hline
\end{tabular}


Table 1. Continued.

\begin{tabular}{|c|c|c|c|c|}
\hline Entry & Ester & Product & $\begin{array}{l}\text { Time } \\
\text { (h) }\end{array}$ & $\begin{array}{l}\text { Yield } \\
(\%)^{b}\end{array}$ \\
\hline 10 & & & $\begin{array}{l}21: 20 \\
(2: 45)\end{array}$ & $\begin{array}{c}61 \\
(87)\end{array}$ \\
\hline 11 & & & $\begin{array}{l}21: 15 \\
(2: 50)\end{array}$ & $\begin{array}{c}60 \\
(85)\end{array}$ \\
\hline 12 & & & $\begin{array}{l}22: 30 \\
(3: 00)\end{array}$ & $\begin{array}{c}60 \\
(85)\end{array}$ \\
\hline 13 & & & $\begin{array}{l}22: 50 \\
(3: 00)\end{array}$ & $\begin{array}{c}55 \\
(80)\end{array}$ \\
\hline 14 & & & $\begin{array}{l}22: 35 \\
(3: 00)\end{array}$ & $\begin{array}{c}50 \\
(87)\end{array}$ \\
\hline 15 & & & $\begin{array}{l}24: 40 \\
(3: 30)\end{array}$ & $\begin{array}{c}50 \\
(76)\end{array}$ \\
\hline 16 & & & $\begin{array}{l}24: 55 \\
(0: 25)\end{array}$ & $\begin{array}{c}50 \\
(78)\end{array}$ \\
\hline
\end{tabular}

${ }^{a}$ In parentheses $C$-alkylation reaction times of barbituric acid in the presence of nano-MOR are given.

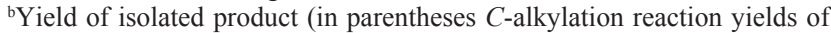
barbituric acid in the presence of nano-MOR are given).

It was observed, from the results in Table 1, that although the reaction occurs without nano-mordenite, the reaction time is very long and the product is obtained in low yield. We believe that the presence of nano-mordenite acts as a media and provides a very effective heterogeneous surface area.

In order to evaluate the role of nano-mordenite in this reaction, we studied the model reaction in the presence of various solvents at $30^{\circ} \mathrm{C}$ (Table 2). As Table 2 indicates, when $\mathrm{K}_{2} \mathrm{CO}_{3}$ was used in presence of nano-MOR, higher yield, as well as shorter reaction time, was observed (Table 2 entry 9).

Table 2. The effect of different solvents upon addition of barbituric acid to ethyl acrylate ${ }^{\mathrm{a}}$

\begin{tabular}{|c|c|c|c|c|}
\hline Entry & Base & Solvent & Time(h) & Yield(\%) \\
\hline 1 & $\mathrm{~K}_{2} \mathrm{CO}_{3}$ & $\mathrm{H}_{2} \mathrm{O}$ & 24 & - \\
\hline 2 & $\mathrm{~K}_{2} \mathrm{CO}_{3}$ & $\mathrm{EtOH}$ & 18 & 25 \\
\hline 3 & $\mathrm{~K}_{2} \mathrm{CO}_{3}$ & $\mathrm{MeOH}$ & 18 & 28 \\
\hline 4 & $\mathrm{~K}_{2} \mathrm{CO}_{3}$ & $\mathrm{CH}_{3} \mathrm{COOEt}$ & 20 & 30 \\
\hline 5 & $\mathrm{~K}_{2} \mathrm{CO}_{3}$ & $\mathrm{CHCl}_{3}$ & 22 & 25 \\
\hline 6 & $\mathrm{~K}_{2} \mathrm{CO}_{3}$ & $\mathrm{CH}_{2} \mathrm{Cl}_{2}$ & 24 & 25 \\
\hline 7 & $\mathrm{~K}_{2} \mathrm{CO}_{3}$ & n-Hexan & 24 & 20 \\
\hline 8 & $\mathrm{~K}_{2} \mathrm{CO}_{3}$ & Solvent-free & 17 & 35 \\
\hline 9 & $\mathrm{~K}_{2} \mathrm{CO}_{3}$ & Mor & 18 & 30 \\
\hline 10 & $\mathrm{~K}_{2} \mathrm{CO}_{3}$ & nano-Mor & 2 & 50 \\
\hline
\end{tabular}

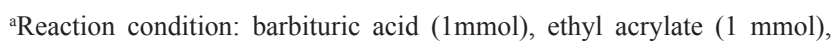
$\mathrm{K}_{2} \mathrm{CO}_{3}(1 \mathrm{mmol})$, at $30^{\circ} \mathrm{C}$.

bIsolated yield.

'Ethyl acrylate $(1 \mathrm{mmol})$, barbituric acid $(1 \mathrm{mmol})$, and $\mathrm{K}_{2} \mathrm{CO}_{3}(1 \mathrm{mmol}) /$ MOR ( 0.5 gr), at $30^{\circ} \mathrm{C}$ under solvent-free conditions.

${ }^{\mathrm{d}}$ Ethyl acrylate $(1 \mathrm{mmol})$, barbituric acid $(1 \mathrm{mmol})$, and $\mathrm{K}_{2} \mathrm{CO}_{3}(1 \mathrm{mmol}) /$ nano-MOR $(0.5 \mathrm{gr})$, at $30{ }^{\circ} \mathrm{C}$ under solvent-free conditions.
In another experiment, we performed the model reaction in the presence of several organic and inorganic bases at $80-90{ }^{\circ} \mathrm{C}$ in the absence of solvent, and the results were summarized in Table 3 . These results showed that supported potassium carbonate on nano-MOR gave the best results and produced the expected product in $90 \%$ yield (Table 3 , entry 6 ).

Table 3. Influence of various bases on the addition of barbituric acid to ethyl acrylate under solvent-free conditions ${ }^{\mathrm{a}}$

\begin{tabular}{|c|c|c|c|c|c|c|}
\hline Entry & 1 & 2 & 3 & 4 & 5 & 6 \\
\hline Base & $\mathrm{Et}_{3} \mathrm{~N}$ & $\mathrm{DABCO}$ & $\mathrm{NaOH}$ & $\mathrm{KOH}$ & $\mathrm{K}_{2} \mathrm{CO}_{3}$ & $\begin{array}{c}\mathrm{K}_{2} \mathrm{CO}_{3} / \\
\text { nano- } \\
\mathrm{MOR}\end{array}$ \\
\hline Time(h) & 24 & 18 & 18 & 20 & 15 & 2 \\
\hline Yield (\%) & - & 25 & 28 & 30 & 57 & 90 \\
\hline
\end{tabular}

a Reaction condition: barbituric acid $(1 \mathrm{mmol})$, ethyl acrylate $(1 \mathrm{mmol})$, base $(1 \mathrm{mmol})$, at $80-90^{\circ} \mathrm{C}$.

bsolated yield.

The recovery and reusability of the catalyst (nano-MOR) was studied on the model reaction. After completion of the reaction the product was extracted by chloroform. Un-dissolved nano-MOR was removed by simple filtration, dried at $200{ }^{\circ} \mathrm{C}$ and reused three times without significant loss of activity.

\section{CONCLUSION}

A convenient, simple and green process has been developed for the $C$-alkylation of barbituric acid using supported $\mathrm{K}_{2} \mathrm{CO}_{3}$ on nano-MOR type of zeolite under solvent-free conditions. The double Michael addition on this substrate was not successful even with excess Michael acceptor addition. This method is very simple, efficient, and environmentally friendly. It was demonstrated that among the organic and inorganic bases, potassium carbonate, as a cheap and green base, effectively catalyses this reaction in the presence of nano-MOR under solvent-free condition.

\section{EXPERIMENTAL}

$\alpha, \beta$-Unsaturated esters were synthesized in our laboratory according to literature procedures. ${ }^{25}$ The progress of the reactions was followed by TLC, using silica gel SILIG/UV 254 plates. Nano mordenite (nano-MOR) was synthesized in our laboratory. ${ }^{26}{ }^{1} \mathrm{H}$ NMR $(400 \mathrm{MHz})$ and ${ }^{13} \mathrm{C}$ NMR $(100 \mathrm{MHz})$ spectra were recorded on a Bruker $300 \mathrm{MHz}$ instrument. FT-IR spectra were recorded on a Perkin-Elmer RX-1 instrument. Mass spectra were recorded on a Shimadzu GC-MS-QP 1000PX. Elemental analysis for C, H, and N was performed using a Heraeus CHN-O-Rapid analyzer. The melting points were determined in open capillaries with a Stuart Melting Point Apparatus, and are uncorrected.

General procedure for addition of barbituric acid to $\alpha, \beta$-unsaturated ester. The nano-MOR is ground together with $\mathrm{K}_{2} \mathrm{CO}_{3}\left(1 \mathrm{mmol} \mathrm{K}_{2} \mathrm{CO}_{3} / 0.5 \mathrm{gr}\right.$ nano-MOR) in a mortar and pestle for 15 minutes. Next, $\alpha, \beta$-unsaturated esters $(1 \mathrm{mmol})$ and barbituric acid $(1 \mathrm{mmol})$ were added to this mixture and kept in the oil bath for the stipulated time (Table 1) at $80-90{ }^{\circ} \mathrm{C}$. The progress of the reaction was monitored by TLC. After completion of reaction the resulting mixture was cooled to room temperature and suspended in chloroform (30 $\mathrm{mL}$ ). Un-dissolved nano-MOR was separated by centrifugation and subsequent washing with chloroform. The chloroform layer was washed with water $(2 \times 10$ $\mathrm{mL}$ ) and dried over anhydrous $\mathrm{Na}_{2} \mathrm{SO}_{4}$ The solvent was removed under reduced pressure and the resulting solid material was recrystallyzed from EtOH.

Physical and spectroscopic data of isolated products. Ethyl 3-(hexahydro-2,4,6-trioxopyrimidin-5-yl)propanoate (2a): White solid, m.p. 98-100 ${ }^{\circ} \mathrm{C}$. FT IR (KBr): n 3378, 3328, 2941, 1732, 1675, 1396, 1378, 1254 , 1193, 1094, 806, $734 \mathrm{~cm}^{-1} .{ }^{1} \mathrm{H}$ NMR (DMSO, TMS, $400 \mathrm{MHz}$ ): $\delta 1.17$ (t, $J=$ $7.15 \mathrm{~Hz}, 3 \mathrm{H}) ; 2.21-2.24(\mathrm{~m}, 4 \mathrm{H}) ; 4.03(\mathrm{q}, J=7.05,2 \mathrm{H}) ; 10.21(\mathrm{~s}, 2 \mathrm{H}) \mathrm{ppm} .{ }^{13} \mathrm{C}$ NMR (DMSO, TMS, $100 \mathrm{MHz}): \delta 14.58 ; 31.26 ; 32.77 ; 60.01 ; 80.33 ; 154.42$; 164.26; $174.50 \mathrm{ppm}$. MS $(70 \mathrm{eV}), \mathrm{m} / \mathrm{e}: 228\left(\mathrm{M}^{+}\right)$. Anal. calcd for $\mathrm{C}_{9} \mathrm{H}_{12} \mathrm{~N}_{2} \mathrm{O}_{5}: \mathrm{C}$, 47.37; H, 5.26; N, 12.28. Found: C, 47.53; H, 5.10; N, 12.56 .

Butyl 3-(hexahydro-2,4,6-trioxopyrimidin-5-yl)propanoate (2b): White solid, m.p. 105-108 ${ }^{\circ} \mathrm{C}$; FT IR (KBr): n 3390, 2961, 1720, 1693, 1475, 1393, $1310,781,550 \mathrm{~cm}^{-1} .{ }^{1} \mathrm{H}$ NMR (DMSO, TMS, $\left.400 \mathrm{MHz}\right): \delta 0.88(\mathrm{t}, J=7.33$ 
$\mathrm{Hz}, 3 \mathrm{H}$ ); 1.31 (sex, $J=7.50 \mathrm{~Hz}, 2 \mathrm{H}$ ), 1.53 (quin, $J=6.96 \mathrm{~Hz}, 2 \mathrm{H}$ ); $2.24-2.38$ $(\mathrm{m}, 4 \mathrm{H}) ; 3.97(\mathrm{t}, J=6.60 \mathrm{~Hz}, 2 \mathrm{H}) ; 9.04(\mathrm{~s}, 2 \mathrm{H}) \mathrm{ppm} .{ }^{13} \mathrm{C}$ NMR (DMSO, TMS, $100 \mathrm{MHz}): \delta 13.58 ; 18.63 ; 19.18 ; 30.26 ; 33.79 ; 63.01 ; 83.33 ; 151.50 ; 164.22$; $173.47 \mathrm{ppm}$. MS $(70 \mathrm{eV}), \mathrm{m} / \mathrm{e}: 256\left(\mathrm{M}^{+}\right)$. Anal. calcd for $\mathrm{C}_{11} \mathrm{H}_{16} \mathrm{~N}_{2} \mathrm{O}_{5}: \mathrm{C}, 51.56$; H, 6.25; N, 10.94. Found: C, 51.81; H, 6.43; N, 10.55 .

Pentyl 3-(hexahydro-2,4,6-trioxopyrimidin-5-yl)propanoate (2c): White solid, m.p. 112-116 ${ }^{\circ} \mathrm{C}$. FT IR (KBr): n 3409, 3248, 2985, 1725, 1574, 1378, 1031, 755, $668 \mathrm{~cm}^{-1} .{ }^{1} \mathrm{H}$ NMR (DMSO, TMS, $400 \mathrm{MHz}$ ): $\delta 0.89$ (t, $J=7.05 \mathrm{~Hz}$, $3 \mathrm{H}) ; 1.28-1.29(\mathrm{~m}, 4 \mathrm{H}) ; 1.56$ (quin, $J=7.00 \mathrm{~Hz}, 2 \mathrm{H}) ; 2.15(\mathrm{q}, J=7.55,2 \mathrm{H})$; $2.40(\mathrm{t}, J=7.40 \mathrm{~Hz}, 2 \mathrm{H}) ; 3.69(\mathrm{t}, J=5.55 \mathrm{~Hz}, 1 \mathrm{H}) ; 3.98(\mathrm{t}, J=6.70 \mathrm{~Hz}, 2 \mathrm{H})$; 11.16 (s, 2H) ppm. ${ }^{13} \mathrm{C}$ NMR (DMSO, TMS, $\left.100 \mathrm{MHz}\right): \delta 13.79 ; 21.72 ; 21.96$; $27.51 ; 27.71 ; 30.70 ; 46.84 ; 63.86 ; 150.84 ; 169.20 ; 172.59$ ppm. MS (70 eV), $\mathrm{m} / \mathrm{e}: 270\left(\mathrm{M}^{+}\right)$. Anal. calcd for $\mathrm{C}_{12} \mathrm{H}_{18} \mathrm{~N}_{2} \mathrm{O}_{5}: \mathrm{C}, 53.33 ; \mathrm{H}, 6.67 ; \mathrm{N}, 10.37$. Found: C, $53.63 ; \mathrm{H}, 6.46 ; \mathrm{N}, 10.21$

Hexyl 3-(hexahydro-2,4,6-trioxopyrimidin-5-yl)propanoate (2d): White solid, m.p. 117-120 ${ }^{\circ} \mathrm{C}$. FT IR (KBr): n 3245, 2930, 1760, 1731, 1176, 1113 , 1039, 754, $546 \mathrm{~cm}^{-1} .{ }^{1} \mathrm{H}$ NMR (DMSO, TMS, $\left.400 \mathrm{MHz}\right): \delta 0.87(\mathrm{t}, J=7.2 \mathrm{~Hz}$, $3 \mathrm{H}$ ); $1.25-1.30(\mathrm{~m}, 6 \mathrm{H}) ; 1.54$ (quin, $J=6.50 \mathrm{~Hz}, 2 \mathrm{H}) ; 2.15(\mathrm{q}, J=6.2 \mathrm{~Hz}, 2 \mathrm{H}$ ); $2.40(\mathrm{t}, J=7.25 \mathrm{~Hz}, 2 \mathrm{H}) ; 3.69(\mathrm{t}, J=5.35 \mathrm{~Hz}, 1 \mathrm{H}) ; 3.98(\mathrm{t}, J=6.55 \mathrm{~Hz}, 2 \mathrm{H})$; $11.16(\mathrm{~s}, 2 \mathrm{H}) \mathrm{ppm} .{ }^{13} \mathrm{C}$ NMR (DMSO, TMS, $\left.100 \mathrm{MHz}\right): \delta 13.83 ; 21.94 ; 24.98 ;$ $27.97 ; 30.70 ; 30.82 ; 46.83 ; 63.87 ; 64.32 ; 150.83 ; 169.89 ; 172.19$ ppm. MS (70 eV), m/e: $284\left(\mathrm{M}^{+}\right)$. Anal. calcd for $\mathrm{C}_{13} \mathrm{H}_{20} \mathrm{~N}_{2} \mathrm{O}_{5}: \mathrm{C}, 54.93 ; \mathrm{H}, 7.04 ; \mathrm{N}, 9.86$. Found: C, 54.75; H, 7.12; N, 9.76.

Octyl 3-(hexahydro-2,4,6-trioxopyrimidin-5-yl)propanoate (2e): White solid, m.p. $115-118^{\circ} \mathrm{C}$. FT IR (KBr): n 3234, 2976, 1760, 1577, 1525, 1393 , $1175,1037,754,546 \mathrm{~cm}^{-1} .{ }^{1} \mathrm{H}$ NMR (DMSO, TMS, $\left.400 \mathrm{MHz}\right): \delta 0.85(\mathrm{t}, J=$ $10.00 \mathrm{~Hz}, 3 \mathrm{H}) ; 1.25-1.26(\mathrm{~m}, 10 \mathrm{H}) ; 1.53$ (quin, $J=10.00 \mathrm{~Hz}, 2 \mathrm{H}) ; 2.08-2.21$ $(\mathrm{m}, 2 \mathrm{H}) ; 2.39(\mathrm{t}, J=6.50 \mathrm{~Hz}, 2 \mathrm{H}) ; 3.98$ (t, $J=7.5 \mathrm{~Hz}, 2 \mathrm{H}) ; 11.09(\mathrm{~s}, 2 \mathrm{H})$ ppm. ${ }^{13} \mathrm{C}$ NMR (DMSO, TMS, $100 \mathrm{MHz}$ ): $\delta 13.95 ; 22.45 ; 23.15 ; 25.19 ; 27.31$; $28.48 ; 30.29 ; 31.37 ; 33.98 ; 47.18 ; 64.37 ; 151.62 ; 169.97 ; 172.25$ ppm. MS (70 eV), m/e: $312\left(\mathrm{M}^{+}\right)$. Anal. calcd for $\mathrm{C}_{15} \mathrm{H}_{24} \mathrm{~N}_{2} \mathrm{O}_{5}: \mathrm{C}, 57.69 ; \mathrm{H}, 7.69 ; \mathrm{N}, 8.97$. Found: C, 57.48; H, 7.55; $\mathrm{N}, 8.78$.

Decyl 3-(hexahydro-2,4,6-trioxopyrimidin-5-yl)propanoate (2f): White solid, m.p. $120-123{ }^{\circ} \mathrm{C}$. FT IR (KBr): n 3256, 2956, 1760, 1733, 1519, 1384, $1106,755,545 \mathrm{~cm}^{-1}$

${ }^{1} \mathrm{H}$ NMR (DMSO ,TMS, $\left.400 \mathrm{MHz}\right): \delta 0.86(\mathrm{t}, J=6.50 \mathrm{~Hz}, 3 \mathrm{H}) ; 1.23-1.27$ (m, 14H); 1.55 (quin, $J=6.65 \mathrm{~Hz}, 2 \mathrm{H}) ; 2.15(\mathrm{q}, J=6.2 \mathrm{~Hz}, 2 \mathrm{H}) ; 2.40(\mathrm{t}, J=$ $7.2 \mathrm{~Hz}, 2 \mathrm{H}) ; 3.69(\mathrm{t}, J=5.35 \mathrm{~Hz}, 1 \mathrm{H}) ; 3.98(\mathrm{t}, J=6.5 \mathrm{~Hz}, 2 \mathrm{H}) ; 11.16(\mathrm{~s}, 2 \mathrm{H})$ ppm. ${ }^{13} \mathrm{C}$ NMR (DMSO, TMS, $100 \mathrm{MHz}$ ): $\delta 13.90 ; 22.05 ; 25.31 ; 28.02 ; 28.62$; $28.88 ; 30.70 ; 31.24 ; 46.83 ; 63.84 ; 150.81 ; 169.88 ; 172.19$ ppm. MS (70 eV) $\mathrm{m} / \mathrm{e}: 340\left(\mathrm{M}^{+}\right)$. Anal. calcd for $\mathrm{C}_{17} \mathrm{H}_{28} \mathrm{~N}_{2} \mathrm{O}_{5}: \mathrm{C}, 60.11 ; \mathrm{H}, 8.24 ; \mathrm{N}, 8.24$. Found: C, $60.21 ; \mathrm{H}, 8.31 ; \mathrm{N}, 8.53$.

2-ethylhexyl 3-(hexahydro-2,4,6-trioxopyrimidin-5-yl)propanoate (2g): White solid, m.p. $116-118^{\circ} \mathrm{C}$. FT IR (KBr): n 3411, 3213, 2962, 1753, 1711 , $1353,1199,807,503,483 \mathrm{~cm}^{-1} .{ }^{1} \mathrm{H}$ NMR (DMSO, TMS, $\left.400 \mathrm{MHz}\right): \delta 0.86(\mathrm{t}$, $J=8.30 \mathrm{~Hz}, 6 \mathrm{H}) ; 1.24-1.31(\mathrm{~m}, 8 \mathrm{H}) ; 2.08-2.13(\mathrm{~m}, 1 \mathrm{H}) ; 2.14(\mathrm{q}, J=7.55 \mathrm{~Hz}$, $2 \mathrm{H}) ; 2.41(\mathrm{t}, J=7.36 \mathrm{~Hz}, 2 \mathrm{H}) ; 3.69(\mathrm{t}, J=5.35 \mathrm{~Hz}, 1 \mathrm{H}) ; 3.90-3.92(\mathrm{dd}, J=1.88$ $\mathrm{Hz}, J=3.52 \mathrm{~Hz}, 2 \mathrm{H}) ; 11.17$ (s, 2H) ppm. ${ }^{13} \mathrm{C}$ NMR (DMSO, TMS, $100 \mathrm{MHz}$ ): $\delta 10.78 ; 13.89 ; 21.94 ; 22.39 ; 23.20 ; 28.31 ; 29.77 ; 30.68 ; 30.76 ; 38.07 ; 46.86$; $65.98 ; 150.87 ; 169.92 ; 172.26$ ppm. MS $(70 \mathrm{eV}), \mathrm{m} / \mathrm{e}: 312\left(\mathrm{M}^{+}\right)$. Anal. calcd for $\mathrm{C}_{15} \mathrm{H}_{24} \mathrm{~N}_{2} \mathrm{O}_{5}: \mathrm{C}, 57.69 ; \mathrm{H}, 7.69 ; \mathrm{N}, 8.97$. Found: C, 57.39; H, 7.61; N, 8.66.

Benzyl 3-(hexahydro-2,4,6-trioxopyrimidin-5-yl)propanoate (2h): White solid, m.p. 148-152 ${ }^{\circ} \mathrm{C}$. FT IR (KBr): n 3422, 3303, 3202, 3076, 1752, 1721, $1365,1195,1043,731,594,506 \mathrm{~cm}^{-1} .{ }^{1} \mathrm{H}$ NMR (DMSO, TMS, $400 \mathrm{MHz}$ ): $\delta$ $2.13(\mathrm{t}, J=6.05 \mathrm{~Hz}, 2 \mathrm{H}) ; 2.46(\mathrm{t}, J=6.50 \mathrm{~Hz}, 2 \mathrm{H}) ; 5.07(\mathrm{~s}, 2 \mathrm{H}) ; 7.31-7.39(\mathrm{~m}$, $5 \mathrm{H}) ; 11.11(\mathrm{~s}, 2 \mathrm{H}) \mathrm{ppm} .{ }^{13} \mathrm{C}$ NMR (DMSO, TMS, $\left.100 \mathrm{MHz}\right): \delta 27.51 ; 34.87$; $65.58 ; 73.22 ; 127.53 ; 127.98 ; 128.47 ; 136.15 ; 150.23 ; 172.32 ; 174.25 \mathrm{ppm}$. MS $(70 \mathrm{eV}), \mathrm{m} / \mathrm{e}: 290\left(\mathrm{M}^{+}\right)$. Anal. calcd for $\mathrm{C}_{14} \mathrm{H}_{14} \mathrm{~N}_{2} \mathrm{O}_{5}: \mathrm{C}, 57.93 ; \mathrm{H}, 4.83 ; \mathrm{N}$, 9.83. Found: C, $57.84 ; \mathrm{H}, 4.69 ; \mathrm{N}, 9.76$.

Diethyl 2-(hexahydro-2,4,6-trioxopyrimidin-5-yl)succinate (3a): White solid, m.p. $135-139^{\circ} \mathrm{C}$. FT IR (KBr): n 3224, 3099, 2988, 1717, 1429, 1351, 1261, 1207, 1029, 796, $529 \mathrm{~cm}^{-1}$; ${ }^{1} \mathrm{H}$ NMR (DMSO, TMS, $400 \mathrm{MHz}$ ): $\delta 1.11$ $(\mathrm{t}, J=8.90 \mathrm{~Hz}, 3 \mathrm{H}) ; 1.21(\mathrm{t}, J=8.90 \mathrm{~Hz}, 3 \mathrm{H}) ; 2.71(\mathrm{dd}, J=10.81 \mathrm{~Hz}, J=7.50$ $\mathrm{Hz}, 1 \mathrm{H}) ; 2.92(\mathrm{dd}, J=10.81 \mathrm{~Hz}, J=8.70 \mathrm{~Hz}, 1 \mathrm{H}) ; 3.82(\mathrm{ddd}, J=7.50 \mathrm{~Hz}, J$ $=8.70 \mathrm{~Hz} J=2.21 \mathrm{~Hz}, 1 \mathrm{H}) ; 3.97(\mathrm{q}, J=8.90 \mathrm{~Hz}, 2 \mathrm{H}) ; 4.05(\mathrm{q}, J=8.90 \mathrm{~Hz}$, $2 \mathrm{H}) ; 4.12(\mathrm{~d}, J=2.21 \mathrm{~Hz}, 1 \mathrm{H}) ; 11.34(\mathrm{~s}, 1 \mathrm{H}) ; 11.39(\mathrm{~s}, 1 \mathrm{H}) \mathrm{ppm} .{ }^{13} \mathrm{C}$ NMR
(DMSO, TMS, $100 \mathrm{MHz}$ ): $\delta 13.99 ; 14.09 ; 34.05 ; 34.05 ; 49.16 ; 61.06 ; 61.14$; $150.68 ; 168.93 ; 169.65 ; 171.34 ; 171.53$ ppm. MS $(70 \mathrm{eV}), \mathrm{m} / \mathrm{e}: 301\left(\mathrm{M}^{+}+1\right)$. Anal. calcd for $\mathrm{C}_{12} \mathrm{H}_{16} \mathrm{~N}_{2} \mathrm{O}_{7}$ : C, 48.12; H, 5.23; N, 9.33. Found: C, 48.21; H, $5.42 ; \mathrm{N}, 9.54$.

Dipropyl 2-(hexahydro-2,4,6-trioxopyrimidin-5-yl)succinate (3b): White solid, m.p. $146-149^{\circ} \mathrm{C}$. FT IR (KBr): n 3226, 3102, 2964, 2940, 2879, 1720 , $1429,1352,1205,802,502 \mathrm{~cm}^{-1}$. ${ }^{1} \mathrm{H}$ NMR (DMSO, TMS, $400 \mathrm{MHz}$ ): $\delta 0.67$ (t, $J=7.41 \mathrm{~Hz}, 3 \mathrm{H}) ; 0.70$ (t, $J=7.42 \mathrm{~Hz}, 3 \mathrm{H}) ; 1.35(\mathrm{~m}, 4 \mathrm{H}) ; 2.71$ (dd, $J=$ $10.23 \mathrm{~Hz}, J=7.93 \mathrm{~Hz}, 1 \mathrm{H}) ; 2.90(\mathrm{dd}, J=10.23 \mathrm{~Hz}, J=6.84 \mathrm{~Hz}, 1 \mathrm{H}) ; 3.58$ (ddd, $J=7.93 \mathrm{~Hz}, J=6.84 \mathrm{~Hz}, J=1.77 \mathrm{~Hz}, 1 \mathrm{H}) ; 3.78(\mathrm{t}, J=6.74 \mathrm{~Hz}, 2 \mathrm{H}$ ); $3.81(\mathrm{t}, J=6.26 \mathrm{~Hz}, 2 \mathrm{H}) ; 3.82(\mathrm{~d}, J=1.77 \mathrm{~Hz}, 1 \mathrm{H}) ; 10.89(\mathrm{~s}, 1 \mathrm{H}) ; 10.94(\mathrm{~s}, 1 \mathrm{H})$ ppm. ${ }^{13} \mathrm{C}$ NMR (DMSO, TMS, $\left.100 \mathrm{MHz}\right): \delta 10.57 ; 10.63 ; 21.93 ; 22.08 ; 33.90$; $33.90 ; 48.72 ; 66.57 ; 67.44 ; 150.83 ; 168.65 ; 169.45 ; 171.80 ; 172.00$ ppm. MS $(70 \mathrm{eV}), \mathrm{m} / \mathrm{e}: 329\left(\mathrm{M}^{+}+1\right)$. Anal. calcd for $\mathrm{C}_{14} \mathrm{H}_{20} \mathrm{~N}_{2} \mathrm{O}_{7}: \mathrm{C}, 51.12 ; \mathrm{H}, 6.10 ; \mathrm{N}$, 8.54. Found: $\mathrm{C}, 51.27 ; \mathrm{H}, 6.25 ; \mathrm{N}, 8.78$

Dibutyl 2-(hexahydro-2,4,6-trioxopyrimidin-5-yl)succinate (3c): White solid, m.p. 141-143 ${ }^{\circ}$ C. FT IR (KBr): n 3223, 3106, 2961, 2936, 2874, 1722, 1428, 1352, 1258, 1202, 804, $502 \mathrm{~cm}^{-1}$. ${ }^{2} \mathrm{H}$ NMR (DMSO, TMS, $400 \mathrm{MHz}$ ): $\delta 0.85(\mathrm{t}, J=7.00 \mathrm{~Hz}, 3 \mathrm{H}) ; 0.90(\mathrm{t}, J=7.20 \mathrm{~Hz}, 3 \mathrm{H}) ; 1.25(\mathrm{~m}, 2 \mathrm{H}) ; 1.35(\mathrm{~m}$, $2 \mathrm{H}) ; 1.42(\mathrm{~m}, 2 \mathrm{H}) ; 1.54(\mathrm{~m}, 2 \mathrm{H}) ; 2.71(\mathrm{dd}, J=11.45 \mathrm{~Hz}, J=6.54 \mathrm{~Hz}, 1 \mathrm{H})$; $2.90(\mathrm{dd}, J=11.45 \mathrm{~Hz}, J=8.51 \mathrm{~Hz}, 1 \mathrm{H}) ; 3.87(\mathrm{ddd}, J=8.51 \mathrm{~Hz}, J=8.51 \mathrm{~Hz}$, $J=1.93 \mathrm{~Hz}, 1 \mathrm{H}) ; 3.96(\mathrm{t}, J=6.55 \mathrm{~Hz}, 2 \mathrm{H}) ; 4(\mathrm{t}, J=6.27 \mathrm{~Hz}, 2 \mathrm{H}) ; 4.15(\mathrm{~d}, J$ $=2.93 \mathrm{~Hz}, 1 \mathrm{H}) ; 11.39(\mathrm{~s}, 1 \mathrm{H}) ; 11.44(\mathrm{~s}, 1 \mathrm{H}) \mathrm{ppm} .{ }^{13} \mathrm{C}$ NMR $(\mathrm{DMSO}, \mathrm{TMS}$, $100 \mathrm{MHz}): \delta 13.50 ; 13.60 ; 18.49 ; 18.63 ; 29.95 ; 30.15 ; 34.13 ; 34.13 ; 49.33$; $63.95 ; 64.74 ; 150.63 ; 168.81 ; 169.60 ; 171.35 ; 171.60 \mathrm{ppm}$. MS $(70 \mathrm{eV}), \mathrm{m} / \mathrm{e}:$ $357\left(\mathrm{M}^{+}+1\right)$. Anal. calcd for $\mathrm{C}_{16} \mathrm{H}_{24} \mathrm{~N}_{2} \mathrm{O}_{7}: \mathrm{C}, 53.93 ; \mathrm{H}, 6.74 ; \mathrm{N}, 7.86$. Found: $\mathrm{C}$, $53.48 ; \mathrm{H}, 6.55 ; \mathrm{N}, 7.78$.

Dipentyl 2-(hexahydro-2,4,6-trioxopyrimidin-5-yl)succinate (3d): White solid, m.p. $123-126{ }^{\circ} \mathrm{C}$. FT IR (KBr): n 3224, 3105, 2961, 2935, 2875, 1732 , $1428,1352,1260,1205,802,502 \mathrm{~cm}^{-1} .{ }^{1} \mathrm{H}$ NMR (DMSO, TMS, $400 \mathrm{MHz}$ ): $\delta 0.82(\mathrm{t}, J=6.54 \mathrm{~Hz}, 3 \mathrm{H}) ; 0.87(\mathrm{t}, J=6.55 \mathrm{~Hz}, 3 \mathrm{H}) ; 1.17(\mathrm{~m}, 8 \mathrm{H}) ; 1.48(\mathrm{~m}$, $2 \mathrm{H}) ; 1.54(\mathrm{~m}, 2 \mathrm{H}) ; 2.71(\mathrm{dd}, J=11.52 \mathrm{~Hz}, J=5.23 \mathrm{~Hz}, 1 \mathrm{H}) ; 2.90(\mathrm{dd}, J=$ $11.52 \mathrm{~Hz}, J=5.71 \mathrm{~Hz}, 1 \mathrm{H}) ; 3.86(\mathrm{ddd}, J=5.71 \mathrm{~Hz}, J=5.23 \mathrm{~Hz} J=2.50 \mathrm{~Hz}$ $1 \mathrm{H}) ; 3.92(\mathrm{t}, J=6.00 \mathrm{~Hz}, 2 \mathrm{H}) ; 4.02(\mathrm{t}, J=5.27 \mathrm{~Hz}, 2 \mathrm{H}) ; 4.15(\mathrm{~d}, J=2.50 \mathrm{~Hz}$, $1 \mathrm{H}) ; 11.41(\mathrm{~s}, 1 \mathrm{H}) ; 11.43(\mathrm{~s}, 1 \mathrm{H}) \mathrm{ppm} .{ }^{13} \mathrm{C}$ NMR (DMSO, TMS, $\left.100 \mathrm{MHz}\right)$ : $\delta 13.77 ; 13.83 ; 21.67 ; 21.76 ; 27.37 ; 27.53 ; 27.56 ; 27.75 ; 34.13 ; 34.13 ; 49.34$; $64.19 ; 64.99 ; 150.57 ; 168.72 ; 169.53 ; 171.29 ; 171.55 \mathrm{ppm}$. MS $(70 \mathrm{eV}), \mathrm{m} / \mathrm{e}:$ $385\left(\mathrm{M}^{+}+1\right)$. Anal. calcd for $\mathrm{C}_{18} \mathrm{H}_{28} \mathrm{~N}_{2} \mathrm{O}_{7}: \mathrm{C}, 56.25 ; \mathrm{H}, 7.29 ; \mathrm{N}, 7.29$. Found: $\mathrm{C}$, $56.38 ; \mathrm{H}, 7.58 ; \mathrm{N}, 7.54$.

Dihexyl 2-(hexahydro-2,4,6-trioxopyrimidin-5-yl)succinate (3e): White solid, m.p. $119-122^{\circ} \mathrm{C}$. FT IR (KBr): n 3226, 3106, 2963, 2935, 2877, 1722 , $1427,1350,1258,1231,1205,800,501 \mathrm{~cm}^{-1} .{ }^{1} \mathrm{H}$ NMR (DMSO, TMS, 400 MHz): $\delta 0.82(\mathrm{t}, J=7.41 \mathrm{~Hz}, 3 \mathrm{H}) ; 0.87(\mathrm{t}, J=7.44 \mathrm{~Hz}, 3 \mathrm{H}) ; 1.07$ (m, 2H); 1.18 $(\mathrm{m}, 2 \mathrm{H}) ; 1.23(\mathrm{~m}, 2 \mathrm{H}) ; 1.31(\mathrm{~m}, 2 \mathrm{H}) ; 1.38(\mathrm{~m}, 2 \mathrm{H}) ; 1.45(\mathrm{~m}, 2 \mathrm{H}) ; 1.54(\mathrm{~m}, 2 \mathrm{H})$; $1.65(\mathrm{~m}, 2 \mathrm{H}) ; 2.71(\mathrm{dd}, J=10.24 \mathrm{~Hz}, J=7.25 \mathrm{~Hz}, 1 \mathrm{H}) ; 2.90(\mathrm{dd}, J=10.24 \mathrm{~Hz}$, $J=8.51 \mathrm{~Hz}, 1 \mathrm{H}) ; 3.90(\mathrm{ddd}, J=7.25 \mathrm{~Hz}, J=8.51 \mathrm{~Hz} J=2.24 \mathrm{~Hz} 1 \mathrm{H}) ; 4.01(\mathrm{t}$, $J=5.91 \mathrm{~Hz}, 2 \mathrm{H}) ; 4.05(\mathrm{t}, J=5.89 \mathrm{~Hz}, 2 \mathrm{H}) ; 4.15(\mathrm{~d}, J=2.24 \mathrm{~Hz}, 1 \mathrm{H}) ; 11.38(\mathrm{~s}$, $1 \mathrm{H}) ; 11.43(\mathrm{~s}, 1 \mathrm{H}) \mathrm{ppm} .{ }^{13} \mathrm{C}$ NMR (DMSO, TMS, $100 \mathrm{MHz}$ ): $\delta$ 11.09; 11.49 ; $13.59 ; 13.91 ; 18.49 ; 18.63 ; 25.16 ; 25.45 ; 29.96 ; 30.15 ; 33.59 ; 34.14 ; 49.34$; $63.95 ; 64.74 ; 150.62 ; 168.98 ; 169.58 ; 171.35 ; 171.61 \mathrm{ppm}$. MS $(70 \mathrm{eV}), \mathrm{m} / \mathrm{e}:$ $413\left(\mathrm{M}^{+}+1\right)$. Anal. calcd for $\mathrm{C}_{20} \mathrm{H}_{32} \mathrm{~N}_{2} \mathrm{O}_{7}: \mathrm{C}, 58.12 ; \mathrm{H}, 7.77 ; \mathrm{N}, 6.80$. Found: $\mathrm{C}$, $58.41 ; \mathrm{H}, 7.81 ; \mathrm{N}, 6.75$.

Diisobutyl 2-(hexahydro-2,4,6-trioxopyrimidin-5-yl)succinate (3f): White solid, m.p. 136- $138^{\circ} \mathrm{C}$. FT IR (KBr): n 3224, 3105, 2961, 2935, 2874, 1721, $1428,1351,1257,1202,804,502 \mathrm{~cm}^{-1} .{ }^{1} \mathrm{H}$ NMR (DMSO, TMS, $400 \mathrm{MHz}$ ): $\delta$ $0.88(\mathrm{~d}, J=6.61 \mathrm{~Hz}, 6 \mathrm{H}) ; 0.95(\mathrm{~d}, J=6.92 \mathrm{~Hz}, 6 \mathrm{H}) ; 2.1(\mathrm{~m}, 2 \mathrm{H}) ; 2.71(\mathrm{dd}, J$ $=11.26 \mathrm{~Hz}, J=7.43 \mathrm{~Hz}, 1 \mathrm{H}) ; 2.90(\mathrm{dd}, J=11.26 \mathrm{~Hz}, J=8.62 \mathrm{~Hz}, 1 \mathrm{H}) ; 3.85$ $(\mathrm{ddd}, J=7.43 \mathrm{~Hz}, J=8.62 \mathrm{~Hz} J=2.19 \mathrm{~Hz} 1 \mathrm{H}) ; 4.01(\mathrm{~d}, J=6.55 \mathrm{~Hz}, 2 \mathrm{H}) ; 4.08$ $(\mathrm{d}, J=6.82 \mathrm{~Hz}, 2 \mathrm{H}) ; 4.15(\mathrm{~d}, J=2.19 \mathrm{~Hz}, 1 \mathrm{H}) ; 11.38(\mathrm{~s}, 1 \mathrm{H}) ; 11.43(\mathrm{~s}, 1 \mathrm{H})$ ppm. ${ }^{13} \mathrm{C}$ NMR (DMSO, TMS, $100 \mathrm{MHz}$ ): $\delta 17.92 ; 18.29 ; 23.93 ; 24.29 ; 34.09$; $34.25 ; 49.16 ; 66.06 ; 66.54 ; 150.68 ; 168.93 ; 169.65 ; 171.34 ; 171.53$ ppm. MS $(70 \mathrm{eV}), \mathrm{m} / \mathrm{e}: 357\left(\mathrm{M}^{+}+1\right)$. Anal. calcd for $\mathrm{C}_{16} \mathrm{H}_{24} \mathrm{~N}_{2} \mathrm{O}_{7}: \mathrm{C}, 53.93 ; \mathrm{H}, 6.74 ; \mathrm{N}$, 7.86. Found: C, $53.81 ; \mathrm{H}, 6.29 ; \mathrm{N}, 7.89$.

Bis(2-methylbutyl) 2-(hexahydro-2,4,6-trioxopyrimidin-5-yl)succinate (3g): White solid, m.p. 116-119 ${ }^{\circ} \mathrm{C}$. FT IR (KBr): n 3227, 3107, 2963, 2935 , $2877,1721,1422,1351,1258,1205,802,501 \mathrm{~cm}^{-1}$. 'H NMR (DMSO, TMS, $400 \mathrm{MHz}): \delta 0.81(\mathrm{t}, J=7.21 \mathrm{~Hz}, 3 \mathrm{H}) ; 0.83(\mathrm{t}, J=7.00 \mathrm{~Hz}, 3 \mathrm{H}) ; 0.85(\mathrm{~d}, J=$ 
$6.25 \mathrm{~Hz}, 3 \mathrm{H}) ; 0.88(\mathrm{~d}, J=6.20 \mathrm{~Hz}, 3 \mathrm{H}) ; 1.11(\mathrm{~m}, 2 \mathrm{H}) ; 1.21(\mathrm{~m}, 2 \mathrm{H}) ; 1.45(\mathrm{~m}$, $1 \mathrm{H}) ; 1.56(\mathrm{~m}, 1 \mathrm{H}) ; 2.71$ (dd, $J=10.22 \mathrm{~Hz}, J=7.65 \mathrm{~Hz}, 1 \mathrm{H}) ; 2.90$ (dd, $J=10.22$ $\mathrm{Hz}, J=7.51 \mathrm{~Hz}, 1 \mathrm{H}) ; 3.81(\mathrm{ddd}, J=7.51 \mathrm{~Hz}, J=7.65 \mathrm{~Hz} J=2.53 \mathrm{~Hz} 1 \mathrm{H})$; $3.85(\mathrm{dd}, J=10.51 \mathrm{~Hz}, J=5.22 \mathrm{~Hz}, 1 \mathrm{H}) ; 3.91(\mathrm{dd}, J=10.51 \mathrm{~Hz}, J=6.2 \mathrm{~Hz}$, $1 \mathrm{H}) ; 3.97$ (dd, $J=10.55 \mathrm{~Hz}, J=6.14 \mathrm{~Hz}, 1 \mathrm{H}) ; 4.05$ (dd, $J=10.55 \mathrm{~Hz}, J=6.14$ $\mathrm{Hz}, 1 \mathrm{H}) ; 4.15(\mathrm{~d}, J=2.53 \mathrm{~Hz}, 1 \mathrm{H}) ; 11.38(\mathrm{~s}, 1 \mathrm{H}) ; 11.45(\mathrm{~s}, 1 \mathrm{H}) \mathrm{ppm} .{ }^{13} \mathrm{C} \mathrm{NMR}$ (DMSO, TMS, $100 \mathrm{MHz}$ ): $\delta 13.58 ; 13.90 ; 18.48 ; 18.62 ; 25.15 ; 25.44 ; 30.14$; $30.89 ; 33.57 ; 34.13 ; 49.32 ; 63.93 ; 64.72 ; 150.61 ; 168.89 ; 169.57 ; 171.33$; $171.59 \mathrm{ppm}$. MS $(70 \mathrm{eV}), \mathrm{m} / \mathrm{e}: 385\left(\mathrm{M}^{+}+1\right)$. Anal. calcd for $\mathrm{C}_{18} \mathrm{H}_{28} \mathrm{~N}_{2} \mathrm{O}_{7}: \mathrm{C}$, 56.25; H, 7.29; N, 7.29. Found: C, 56.45; H, 7.69; N, 7.78.

Diisopentyl 2-(hexahydro-2,4,6-trioxopyrimidin-5-yl)succinate (3h): White solid, m.p. $135-137^{\circ} \mathrm{C}$. FT IR (KBr): n 3227, 3108, 2960, 2872, 1720 , 1428, 1352, 1258, 1206, 804, $502 \mathrm{~cm}^{-1}$. ${ }^{1} \mathrm{H}$ NMR (DMSO, TMS, $400 \mathrm{MHz}$ ): $\delta 0.83(\mathrm{~d}, J=6.54 \mathrm{~Hz}, 6 \mathrm{H}) ; 0.89(\mathrm{~d}, J=6.83 \mathrm{~Hz}, 6 \mathrm{H}) ; 1.36(\mathrm{~m}, 2 \mathrm{H}) ; 1.48(\mathrm{~m}$, $2 \mathrm{H}) ; 1.58(\mathrm{~m}, 1 \mathrm{H}) ; 1.65(\mathrm{~m}, 1 \mathrm{H}) ; 2.71(\mathrm{dd}, J=11.61 \mathrm{~Hz}, J=5.22 \mathrm{~Hz}, 1 \mathrm{H})$; $2.91(\mathrm{dd}, J=11.61 \mathrm{~Hz}, J=8.62 \mathrm{~Hz}, 1 \mathrm{H}) ; 3.81(\mathrm{ddd}, J=8.62 \mathrm{~Hz}, J=5.22 \mathrm{~Hz}$, $J=2.51 \mathrm{~Hz}, 1 \mathrm{H}) ; 3.98(\mathrm{t}, J=6.54 \mathrm{~Hz}, 2 \mathrm{H}) ; 4.04(\mathrm{t}, J=6.51 \mathrm{~Hz}, 2 \mathrm{H}) ;, 4.15(\mathrm{~d}$, $J=2.51 \mathrm{~Hz}, 1 \mathrm{H}) ; 11.38(\mathrm{~s}, 1 \mathrm{H}) ; 11.43(\mathrm{~s}, 1 \mathrm{H}) \mathrm{ppm} .{ }^{13} \mathrm{C}$ NMR (DMSO, TMS, $100 \mathrm{MHz}): \delta 22.11 ; 22.25 ; 24.18 ; 24.49 ; 33.54 ; 34.08 ; 36.56 ; 36.78 ; 49.32$; $62.67 ; 63.37 ; 150.52 ; 168.67 ; 169.47 ; 171.22 ; 171.51 \mathrm{ppm} . \mathrm{MS}(70 \mathrm{eV}), \mathrm{m} / \mathrm{e}:$ $385\left(\mathrm{M}^{+}+1\right)$. Anal. calcd for $\mathrm{C}_{18} \mathrm{H}_{28} \mathrm{~N}_{2} \mathrm{O}_{7}: \mathrm{C}, 56.25 ; \mathrm{H}, 7.29 ; \mathrm{N}, 7.29$. Found: $\mathrm{C}$, $56.73 ; \mathrm{H}, 7.43 ; \mathrm{N}, 7.60$.

\section{ACKNOWLEDGMENT}

Authors are grateful to, University of Mohaghegh Ardabili for the financial support and the laboratories of Tehran University for the product analysis.

\section{REFERENCES}

1.- $\quad$ M. Meusel, A. Ambrozak, T. K. Hecker, M. Gutschow, J. Org. Chem. 68, 4684, (2003).

2.- H. Brunner, K.P. Ittner, D. Lunz, S. Schmatlock, T, Schmidt, M. Zabel, Eur. J. Org. Chem. 855, (2003).

3.- M. E. Wolff, (Ed.), Burgers Medicinal Chemistry and Drug Discovery, John Wiley, 1997.
4.- R. Rastaldo, C. Penna, P. Pagliaro, Life Sci. 69, 729, (2001).

5.- $\quad$ S. P. Aiken, W. M. Brown, Front. Biosci. 5, 124, (2000).

6.- $\quad$ E. Ghansah, D. S. Weiss, Neuropharmacology 40, 327, (2001).

7.- B. Levine, Princ. Forensic Toxicol. 185, (1999).

8.- J. T. Pinhey, B. A. Rowe, Tetrahedron Lett. 21, 965, (1980).

9.- R. R. Nadendla, Principle of Organic Medicinal Chemistry, New Age International (p) Limited, Pubishers, New Dehli, 2005.

10.- R. N. Westhorpe, C. Ball, Int. Congress Series 1242, 57, (2002).

11.- B. M. Trost, G. Schroeder, J. Org. Chem. 65, 1569, (2000).

12.- J. T. Bojarski, J. L. Mokrosz, H. J. Barton, H. J. Paluchowska, Adv. Heterocyclic Chem. 38, 229, (1985).

13.- W. J. Doran, Med. Chem. 4, 1, (1959).

14.- A. Goth, Medical Pharmacology, $4^{\text {th }}$ ed., The Mosby Company, St. Louis, MN, 1968.

15.- C. Weigand, G. Hilgetag, Preparative Organic Chemistry, John Wiley \& Sons, New York, 1972.

16.- A. I. Vogel, Principal of Organic Chemistry, $3^{\text {rd }}$ ed, John Wiley \& Sons, New York, 1966.

17.- K. A. Krasnov, V. I. Slesarev, Z. L. Artemeva, Zh. Orga. Khim. 25, 1553 , (1989).

18.- A. N. Isaev, K. Y. Burshtein, N. N. Sukhanov, L. A. Yanovskaya, Izv. Akad. Nauk. SSSR. Ser. Khim. 918, (1988).

19.- G. H. Imanzadeh, A. R. Hajipour, S. E. Mallakpour, Synth. Commun. 33, $735,(2003)$

20.- A. R. Hajipour, Mallakpour, S. E. G. H. Imanzadeh, Chemistry Lett. 99, (1999).

21.- A. R. Hajipour, S. E. Mallakpour, G. H. Imanzadeh, J. Chem. Research(s) 228, (1999)

22.- A. N. Osman, M. M. Kandeel, M. M. Said, E. M. Ahmed, Indian J. Chem. 35B 1073, (1996).

23.- M. G. Ahmed, U. K. R. Romman, S. M. Ahmed, K. Akhter, M. E. Halim, M. Salauddin, Bangladesh J. Sci. Ind. Res. 41, 119, (2006).

24.- H. H. Otto, J. Triepel, Leibigs Ann. Chem. 1982, (1967).

25.- A. Vogel, Vogel's Practical Organic Chemistry, $4^{\text {th }}$ ed., Longman Press: London, 1978.

26.- P. Sharm, P. Rajaram, R. Tomar, J. Colloid Interf. Sci. 325, 547, (2008). 